\title{
Correction to: Topology optimization applied to the design of 2D swirl flow devices
}

\author{
Diego Hayashi Alonso ${ }^{1}$ - Luis Fernando Nogueira de Sá ${ }^{1}$ Juan Sergio Romero Saenz ${ }^{2}$ Emílio Carlos Nelli Silva ${ }^{1}$
}

Published online: 13 November 2018

(C) Springer-Verlag GmbH Germany, part of Springer Nature 2018

\section{Correction to: Structural and Multidisciplinary Opti- mization}

https://doi.org/10.1007/s00158-018-2078-0

The original version of this article contains correction that was not properly implemented. The author would like to correct using the updated corrections indicated as follows:

Abstract, line 10 - The text "An interior point optimization (IPOPT) algorithm" should read as "An interior point optimization algorithm (IPOPT)".

Page 2, 3rd paragraph, line 10 - The text "Some examples of fluid devices that can be designed by such models are hydrocyclones, some [...]" should read as "Some examples of fluid devices that can be designed by such model are hydrocyclones, some [...]".

Page 2, 6th paragraph - The text "This paper is organized as follows: in Section 2, the flow [...]" should read as "This paper is organized as follows. In Section 2, the flow [...]".

All occurences - The text "Navier-Stokes equations for a rotating reference frame" should read as italic "NavierStokes equations for a rotating reference frame”.

Page 2, section 2.3, 2nd paragraph, line 2- The text "(3) and (4) in (2)," should read as "equations (3) and (4) in equation (2),"

Page 3, 1st paragraph - The text "force $(-2 \rho(\omega \wedge \boldsymbol{v}))$ " should read as "force $(-2 \rho(\boldsymbol{\omega} \wedge \boldsymbol{v}))$ ".

Page 3, 2nd paragraph - The text "continuity equation for a rotating reference frame" should read as italic "continuity equation for a rotating reference frame".

The online version of the original article can be found at https://doi.org/10.1007/s00158-018-2078-0.

Emílio Carlos Nelli Silva

ecnsilva@usp.br

1 Department of Mechatronics and Mechanical Systems Engineering, Polytechnic School of the University of São Paulo, São Paulo, SP, Brazil

2 Department of Mechanical Engineering, Federal University of Espírito Santo, Espírito Santo, ES, Brazil
Page 5, section 4.4, line 2 - The text "the weak form $F((v, p), \alpha)=0$ (16) can be obtained by the" should read as "the weak form $F((v, p), \alpha)=0$ (equation (16)) can be obtained by the".

Page 5, 1st paragraph in section 5, line 6-7 - The text “(IPOPT Wächter and Biegler 2006) ....” should read as "IPOPT (Wächter and Biegler 2006) ...."

Page 5, 1st paragraph in section 5, line 7 - The text "The solution to the finite element method is performed" should read as "The solution to the Finite Element Method is performed".

Pag 5, 1st paragraph in section 5, line 9-10 - The text "multifrontal massively parallel sparse direct solver" should read as "MUltifrontal Massively Parallel sparse direct Solver".

Page 6, 1st paragraph, line 3-The text "and sensibilities, the MMGS (millimeters-grams-seconds)" should read as "and sensibilities, the MMGS (Millimeters-GramsSeconds)".

Page 6, 2nd paragraph, line 5 - The text "for the optimality error of the IPOPT barrier problem that" should read as "for the optimality error of the IPOPT barrier problem, that".

Page 6, 5th paragraph - The text "In the following examples, the letter $n$ is used to denote rotation in revolutions per minute, and the greek letter $\omega$ is used to denote rotation in radian per second" should read as "In the following examples, the letter $n$ is used to denote rotation in rpm, and the greek letter $\omega$ is used to denote rotation in $\mathrm{rad} / \mathrm{s}$.".

Page 7, 5th paragraph - The text "In the material model, $\kappa_{\min }$ is chosen as $0 \mathrm{~kg} /\left(\mathrm{m}^{3} / \mathrm{s}\right)$ for all examples." should read as "In the material model, $\kappa_{\min }$ is chosen as $0 \mathrm{~kg} /\left(\mathrm{m}^{3} / \mathrm{s}\right)$ for all examples."

All occurrences - The text "12,800" should read as "12800".

Page 8, 1st paragraph, line 7 - The text "corresponds to the post-processed topology (22)." should read as "corresponds to the post-processed topology (equation (22)).".

All occurrences - The text "Measure of non-discreteness" and "measure of non-discreteness" should read as "Measure of Non-Discreteness". 
All occurrences - The text "value shown corresponds to the post-processed topology (22)." should read as "value shown corresponds to the post-processed topology (equation (22))."

Page 13, 4th paragraph, line 3 - The text "totaling $8131 \sim$ 16,181 nodes and 16,000 32,000" should read as "totaling $8131 \sim 16181$ nodes and $16000 \sim 32000 "$.

Page 18, conclusion section, line 6 - The text "This formulation avoids the need of 3D models that have high computational cost to design these devices." should read as "This formulation avoids the need of $3 D$ models, that have high computational cost, to design these devices."

Page 18, conclusion section, 4th paragraph - The text "As future work, the use of the 2D swirl flow model in specific applications is suggested such as in pump/turbine design, the use of non-Newtonian fluid flows, and turbulence models." should read as "As future work, it is suggested that the 2D swirl flow model is used in specific applications such as in pump/turbine design, the use of non-Newtonian fluid flows, and turbulence models."

Page 19, appendix B, lines 4-5 - The text "for the case of a 2D (not 2D swirl) flow is shown in Barth and Carey 2007)." should read as "for the case of a $2 \mathrm{D}$ (not 2D swirl) flow is shown in Barth and Carey (2007)."

Page 19, appendix B - The text "By substituting equation (33) in the continuity equation (7)" should read as "By substituting equation (33) in the continuity equation (equation (7))".

Page 19, appendix B, below equation (42) - The text "its normal vector $\mathbf{n}\left(k_{m}=-\nabla \bullet \mathbf{n}\right.$ Weatherburn 1955)" should read as "its normal vector $\mathbf{n}\left(k_{m}=-\nabla \bullet \mathbf{n}\right.$ (Weatherburn 1955))".

Page 19, appendix B - The text "Therefore, by substituting equation (43) in the equation for the normal stress (35)," should read as "Therefore, by substituting equation (43) in the equation for the normal stress (equation (35),".

All occurrences - The text " $\mathbf{J}_{F}$ " should read as italic " $\boldsymbol{J}_{F}$ ".

The names/fractions in equations (25), (26), (27), (28), (29), (30), (55), (57), (61), (63), (73), (77), (82a), (82b), (82c), (82d), (83a), (83b), (83c), (84a), (84c) and (84d) should have the same size as they have in equation (58) as shown below:

$$
\begin{aligned}
H & =\frac{1}{Q}\left[\int_{\Gamma_{\text {in }}}\left(\frac{p}{\rho g}+\frac{v_{\mathrm{abs}}^{2}}{2 g}+h_{z}\right) \boldsymbol{v} \bullet \boldsymbol{n} 2 \pi r d \Gamma\right. \\
& \left.+\int_{\Gamma_{\text {out }}}\left(\frac{p}{\rho g}+\frac{v_{\mathrm{abs}}^{2}}{2 g}+h_{z}\right) \boldsymbol{v} \bullet \boldsymbol{n} 2 \pi r d \Gamma\right] \\
R_{c} & =\int_{\Omega}\left[\frac{\partial v_{r}}{\partial r}+\frac{v_{r}}{r}+\frac{\partial v_{z}}{\partial z}\right] \boldsymbol{w}_{p} r d \Omega
\end{aligned}
$$

$$
\begin{aligned}
R_{m, r} & =\int_{\Omega}\left[\rho\left(v_{r} \frac{\partial v_{r}}{\partial r}+v_{z} \frac{\partial v_{r}}{\partial z}-\frac{v_{\theta}^{2}}{r}\right) \boldsymbol{w}_{v, r}\right. \\
& +\left(-p+2 \mu \frac{\partial v_{r}}{\partial r}\right) \frac{\partial \boldsymbol{w}_{v, r}}{\partial r} \\
& +\left(-p+2 \mu \frac{v_{r}}{r}\right) \frac{\boldsymbol{w}_{v, r}}{r}+\mu\left(\frac{\partial v_{z}}{\partial r}+\frac{\partial v_{r}}{\partial z}\right) \frac{\partial \boldsymbol{w}_{v, r}}{\partial z} \\
& \left.-\rho f_{r} \boldsymbol{w}_{v, r}-2 \rho \omega_{0} v_{\theta} \boldsymbol{w}_{v, r}\right] r d \Omega \\
& -\int_{\Omega} \rho \omega_{0}^{2} r^{2} \boldsymbol{w}_{v, r} d \Omega-\oint_{\Gamma}(\boldsymbol{T} \bullet \boldsymbol{n})_{r} \boldsymbol{w}_{v, r} r d \Gamma \\
& +\int_{\Omega} \kappa(\alpha) v_{\text {mat }, r} \boldsymbol{w}_{v, r} r d \Omega
\end{aligned}
$$

$$
\begin{aligned}
R_{m, \theta} & =\int_{\Omega}\left[\rho\left(v_{r} \frac{\partial v_{\theta}}{\partial r}+v_{z} \frac{\partial v_{\theta}}{\partial z}+\frac{v_{\theta} \cdot v_{r}}{r}\right) \boldsymbol{w}_{v, \theta}\right. \\
& +\mu\left(\frac{\partial v_{\theta}}{\partial r}-\frac{v_{\theta}}{r}\right)\left(\frac{\partial \boldsymbol{w}_{v, \theta}}{\partial r}-\frac{\boldsymbol{w}_{v, \theta}}{r}\right) \\
& \left.+\mu \frac{\partial v_{\theta}}{\partial z} \frac{\partial \boldsymbol{w}_{v, \theta}}{\partial z}-\rho f_{\theta} \boldsymbol{w}_{v, \theta}+2 \rho \omega_{0} v_{r} \boldsymbol{w}_{v, \theta}\right] r d \Omega \\
& -\oint_{\Gamma}(\boldsymbol{T} \bullet \boldsymbol{n})_{\theta} \boldsymbol{w}_{v, \theta} r d \Gamma+\int_{\Omega} \kappa(\alpha) v_{\mathrm{mat}, \theta} \boldsymbol{w}_{v, \theta} r d \Omega
\end{aligned}
$$

$$
\begin{aligned}
R_{m, z} & =\int_{\Omega}\left[\rho\left(v_{r} \frac{\partial v_{z}}{\partial r}+v_{z} \frac{\partial v_{z}}{\partial z}\right) \boldsymbol{w}_{v, z}\right. \\
& +\mu\left(\frac{\partial v_{z}}{\partial r}+\frac{\partial v_{r}}{\partial z}\right) \frac{\partial \boldsymbol{w}_{v, z}}{\partial r} \\
& \left.+\left(-p+2 \mu \frac{\partial v_{z}}{\partial z}\right) \frac{\partial \boldsymbol{w}_{v, z}}{\partial z}-\rho f_{z} \boldsymbol{w}_{v, z}\right] r d \Omega \\
& -\oint_{\Gamma}(\boldsymbol{T} \bullet \boldsymbol{n})_{z} \boldsymbol{w}_{v, z} r d \Gamma+\int_{\Omega} \kappa(\alpha) v_{\text {mat }, z} \boldsymbol{w}_{v, z} r d \Omega
\end{aligned}
$$

$$
\boldsymbol{T}=\left[\begin{array}{ccc}
-p+2 \mu \frac{\partial v_{r}}{\partial r} & \mu\left(-\frac{v_{\theta}}{r}+\frac{\partial v_{\theta}}{\partial r}\right) & \mu\left(\frac{\partial v_{z}}{\partial r}+\frac{\partial v_{r}}{\partial z}\right) \\
\mu\left(-\frac{v_{\theta}}{r}+\frac{\partial v_{\theta}}{\partial r}\right) & -p+2 \mu \frac{v_{r}}{r} & \mu \frac{\partial v_{\theta}}{\partial z} \\
\mu\left(\frac{\partial v_{z}}{\partial r}+\frac{\partial v_{r}}{\partial z}\right) & \mu \frac{\partial v_{\theta}}{\partial z} & -p+2 \mu \frac{\partial v_{z}}{\partial z}
\end{array}\right]
$$

$$
\begin{aligned}
\boldsymbol{K}_{G l} \rightarrow K_{G 1, i, j} & =\int_{\Omega}\left(2 \mu \frac{\partial \phi_{i}}{\partial r} \frac{\partial \phi_{j}}{\partial r}+2 \mu \frac{\phi_{i} \phi_{j}}{r^{2}}\right. \\
& \left.+\mu \frac{\partial \phi_{i}}{\partial z} \frac{\partial \phi_{j}}{\partial z}\right) r d \Omega
\end{aligned}
$$

$$
\begin{aligned}
\boldsymbol{K}_{G 3} \rightarrow K_{G 3, i, j} & =\int_{\Omega}\left(\mu\left(\frac{\partial \phi_{i}}{\partial r}-\frac{\phi_{i}}{r}\right)\left(\frac{\partial \phi_{j}}{\partial r}-\frac{\phi_{j}}{r}\right)\right. \\
& \left.+\mu \frac{\partial \phi_{i}}{\partial z} \frac{\partial \phi_{j}}{\partial z}\right) r d \Omega
\end{aligned}
$$




$$
\begin{aligned}
\boldsymbol{C}_{G l}(z) \rightarrow C_{G l, i, j}=\int_{\Omega} \rho & \left(v_{r, j} \phi_{i} \frac{\partial \phi_{j}}{\partial r}+\right. \\
& \left.v_{z, j} \frac{\partial \phi_{i}}{\partial z}\right) \phi_{j} r d \Omega
\end{aligned}
$$

$$
\begin{aligned}
& \boldsymbol{C}_{G 3}(\boldsymbol{z}) \rightarrow C_{G 3, i, j}=\int_{\Omega} \rho\left(v_{\theta, j} \phi_{i} \frac{\partial \phi_{j}}{\partial r}+\right. \\
&\left.v_{\theta, j} \frac{\phi_{i} \phi_{j}}{r}\right) \phi_{j} r d \Omega
\end{aligned}
$$

$$
\begin{aligned}
\boldsymbol{K}_{V 3} \rightarrow K_{V 3, i, j}=\int_{\Omega} & \left(\frac{\partial \phi_{i}}{\partial r}+\frac{\phi_{i}}{r}\right) \\
& \left(\frac{\partial \phi_{j}}{\partial r}+\frac{\phi_{j}}{r}\right) 2 \pi r d \Omega
\end{aligned}
$$

$$
\begin{aligned}
\boldsymbol{K}_{3} \rightarrow K_{3, i, j}=\int_{\Omega} \mu & \left(\frac{\partial \phi_{i}}{\partial r}-\frac{\phi_{i}}{r}\right) \\
& \left(\frac{\partial \phi_{j}}{\partial r}-\frac{\phi_{j}}{r}\right) 2 \pi r d \Omega
\end{aligned}
$$

$$
\begin{aligned}
\frac{\partial \boldsymbol{R}_{m, r}}{\partial \boldsymbol{v}_{r}} \rightarrow \frac{\partial \hat{R}_{m, i, r}}{\partial v_{r, j}} & =\int_{\Omega}\left[\rho \left(v_{r} \frac{\partial \phi_{j}}{\partial r}+\frac{\partial \phi_{j}}{\partial r} \frac{\partial v_{r}}{\partial r}\right.\right. \\
& \left.+v_{z} \frac{\partial \phi_{j}}{\partial z}\right) \phi_{i} \\
& +\left(2 \mu \frac{\partial \phi_{j}}{\partial r}\right) \frac{\partial \phi_{i}}{\partial r}+\left(2 \mu \frac{\phi_{j}}{r}\right) \frac{\phi_{i}}{r} \\
& \left.+\mu\left(\frac{\partial \phi_{j}}{\partial z}\right) \frac{\partial \phi_{i}}{\partial z}\right] r d \Omega \\
& -\oint_{\Gamma} \frac{\partial(\boldsymbol{T} \bullet \boldsymbol{n})_{r}}{\partial v_{r, j}} \phi_{i} r d \Gamma \\
& +\int_{\Omega} \kappa(\alpha) \phi_{j} \phi_{i} r d \Omega
\end{aligned}
$$

$$
\begin{aligned}
\frac{\partial \boldsymbol{R}_{m, r}}{\partial \boldsymbol{v}_{\theta}} \rightarrow \frac{\partial \hat{R}_{m, i, r}}{\partial v_{\theta, j}} & =\int_{\Omega}\left[\rho\left(-2 \frac{v_{\theta}}{r} \phi_{j}\right) \phi_{i}\right. \\
& \left.-2 \rho \omega_{0} \phi_{j} \phi_{i}\right] r d \Omega \\
& -\oint_{\Gamma} \frac{\partial(\boldsymbol{T} \bullet \boldsymbol{n})_{r}}{\partial v_{\theta, j}} \phi_{i} r d \Gamma
\end{aligned}
$$

$$
\begin{aligned}
\frac{\partial \boldsymbol{R}_{m, r}}{\partial \boldsymbol{v}_{z}} \rightarrow \frac{\partial \hat{R}_{m, i, r}}{\partial v_{z, j}} & =\int_{\Omega}\left[\rho \frac{\partial v_{r}}{\partial z} \phi_{j} \phi_{i}+\mu \frac{\partial \phi_{j}}{\partial r} \frac{\partial \phi_{i}}{\partial z}\right] r d \Omega \\
& -\oint_{\Gamma} \frac{\partial(\boldsymbol{T} \bullet \boldsymbol{n})_{r}}{\partial v_{z, j}} \phi_{i} r d \Gamma
\end{aligned}
$$

$$
\begin{aligned}
\frac{\partial \boldsymbol{R}_{m, r}}{\partial \boldsymbol{p}} \rightarrow \frac{\partial \hat{R}_{m, i, r}}{\partial p_{j}} & =\int_{\Omega}\left[-\chi_{j} \frac{\partial \phi_{i}}{\partial r}-\chi_{j} \frac{\phi_{i}}{r}\right] r d \Omega \\
& -\oint_{\Gamma} \frac{\partial(\boldsymbol{T} \bullet \boldsymbol{n})_{r}}{\partial p_{j}} \phi_{i} r d \Gamma
\end{aligned}
$$

$$
\begin{aligned}
\frac{\partial \boldsymbol{R}_{m, \theta}}{\partial \boldsymbol{v}_{r}} \rightarrow \frac{\partial \hat{R}_{m, i, \theta}}{\partial v_{r, j}} & =\int_{\Omega}\left[\rho\left(\phi_{j} \frac{\partial v_{\theta}}{\partial r}+\frac{v_{\theta} \phi_{j}}{r}\right) \phi_{i}\right. \\
& \left.+2 \rho \omega_{0} v_{r} \phi_{i}\right] r d \Omega \\
& -\oint_{\Gamma} \frac{\partial(\boldsymbol{T} \bullet \boldsymbol{n})_{\theta}}{\partial v_{r, j}} \phi_{i} r d \Gamma
\end{aligned}
$$

$$
\begin{aligned}
\frac{\partial \boldsymbol{R}_{m, \theta}}{\partial \boldsymbol{v}_{\theta}} \rightarrow \frac{\partial \hat{R}_{m, i, \theta}}{\partial v_{\theta, j}} & =\int_{\Omega}\left[\rho \left(v_{r} \frac{\partial \phi_{j}}{\partial r}+v_{z} \frac{\partial \phi_{j}}{\partial z}\right.\right. \\
& \left.+\frac{\phi_{j} v_{r}}{r}\right) \phi_{i} \\
& +\mu\left(\frac{\partial \phi_{j}}{\partial r}-\frac{\phi_{j}}{r}\right)\left(\frac{\partial \phi_{i}}{\partial r}-\frac{\phi_{i}}{r}\right) \\
& \left.+\mu \frac{\partial \phi_{j}}{\partial z} \frac{\partial \phi_{i}}{\partial z}\right] r d \Omega \\
& -\oint_{\Gamma} \frac{\partial(\boldsymbol{T} \bullet \boldsymbol{n})_{\theta}}{\partial v_{\theta, j}} \phi_{i} r d \Gamma \\
& +\int_{\Omega} \kappa(\alpha) \phi_{j} \phi_{i} r d \Omega
\end{aligned}
$$

$$
\begin{aligned}
\frac{\partial \boldsymbol{R}_{m, \theta}}{\partial \boldsymbol{v}_{z}} \rightarrow \frac{\partial \hat{R}_{m, i, \theta}}{\partial v_{z, j}} & =\int_{\Omega}\left[\rho\left(\phi_{j} \frac{\partial v_{\theta}}{\partial z}\right) \phi_{i}\right] r d \Omega \\
& -\oint_{\Gamma} \frac{\partial(\boldsymbol{T} \bullet \boldsymbol{n})_{\theta}}{\partial v_{z, j}} \phi_{i} r d \Gamma \\
\frac{\partial \boldsymbol{R}_{m, z}}{\partial \boldsymbol{v}_{r}} \rightarrow \frac{\partial \hat{R}_{m, i, z}}{\partial v_{r, j}} & =\int_{\Omega}\left[\rho\left(\phi_{j} \frac{\partial v_{z}}{\partial r}\right) \phi_{i}\right. \\
& \left.+\mu\left(\frac{\partial \phi_{j}}{\partial z}\right) \frac{\partial \phi_{i}}{\partial r}\right] r d \Omega \\
& -\oint_{\Gamma} \frac{\partial(\boldsymbol{T} \bullet \boldsymbol{n})_{z}}{\partial v_{r, j}} \phi_{i} r d \Gamma
\end{aligned}
$$

$$
\begin{aligned}
\frac{\partial \boldsymbol{R}_{m, z}}{\partial \boldsymbol{v}_{z}} \rightarrow \frac{\partial \hat{R}_{m, i, z}}{\partial v_{z, j}} & =\int_{\Omega}\left[\rho \left(v_{r} \frac{\partial \phi_{j}}{\partial r}+v_{z} \frac{\partial \phi_{j}}{\partial z}\right.\right. \\
& \left.+\phi_{j} \frac{\partial v_{z}}{\partial z}\right) \phi_{i}+\mu\left(\frac{\partial \phi_{j}}{\partial r}\right) \frac{\partial \phi_{i}}{\partial r} \\
& \left.+\left(2 \mu \frac{\partial \phi_{j}}{\partial z}\right) \frac{\partial \phi_{i}}{\partial z}\right] r d \Omega \\
& -\oint_{\Gamma} \frac{\partial(\boldsymbol{T} \bullet \boldsymbol{n})_{z}}{\partial v_{z, j}} \phi_{i} r d \Gamma \\
& +\int_{\Omega} \kappa(\alpha) \phi_{j} \phi_{i} r d \Omega
\end{aligned}
$$

$$
\begin{aligned}
\frac{\partial \boldsymbol{R}_{m, z}}{\partial \boldsymbol{p}} \rightarrow \frac{\partial \hat{R}_{m, i, z}}{\partial p_{j}} & =\int_{\Omega}\left[-\chi_{j} \frac{\partial \phi_{i}}{\partial z}\right] r d \Omega \\
& -\oint_{\Gamma} \frac{\partial(\boldsymbol{T} \bullet \boldsymbol{n})_{z}}{\partial p_{j}} \phi_{i} r d \Gamma
\end{aligned}
$$


Page 21, section C. 3 - The text "the weak form $\mathbf{F}(\mathbf{z}, \alpha)=$ 0 (48) can be obtained by the" should read as "the weak form $\mathbf{F}(\mathbf{z}, \alpha)=\mathbf{0}$ (equation (48)) can be obtained by the".

Page 22, The first $\Gamma$ letter of equation (83b) (in its 5 th line) should be in subscript the same as equation (83a) as shown below:

$$
\oint_{\Gamma} \frac{\partial(\boldsymbol{T} \bullet \boldsymbol{n})_{\theta}}{\partial v_{\theta, j}} \phi_{i} r d \Gamma
$$

Page 22 (equations in the end of the left column) - The text "(from (50))", "(from (48))" and "(from (50))" should read as "(from equation (50))", "(from equation (48))" and "(from equation (50))".

Page 22, appendix E - The text "in the equations of Appendix A (26) to (29)" should read as "in the equations of Appendix A (equations (26) to (29))".

Page 23 - The line integral of equations (84a) and (84c) should be counter clockwise. As shown below:

$\oint_{\Gamma}$

Publisher's Note Springer Nature remains neutral with regard to jurisdictional claims in published maps and institutional affiliations. 